Article

\title{
Intelligent Interaction with Virtual Geographical Environments Based on Geographic Knowledge Graph
}

\author{
Bingchuan Jiang ${ }^{1,2}$, Liheng Tan ${ }^{1, *}$, Yan Ren ${ }^{1}$ and Feng Li ${ }^{1}$ \\ 1 Strategic Support Force Information Engineering University, Zhengzhou 450001, China; \\ jbc021@163.com (B.J.); qingyuliuer@163.com (Y.R.); jueyun1020@126.com (F.L.) \\ 2 State Key Laboratory of Geo-information Engineering, Xi'an 750054, China \\ * Correspondence: tanliheng_123@163.com; Tel.: +86-371-8162-3292
}

Received: 29 July 2019; Accepted: 16 September 2019; Published: 24 September 2019

\begin{abstract}
The core of intelligent virtual geographical environments (VGEs) is the formal expression of geographic knowledge. Its purpose is to transform the data, information, and scenes of a virtual geographic environment into "knowledge" that can be recognized by computer, so that the computer can understand the virtual geographic environment more easily. A geographic knowledge graph (GeoKG) is a large-scale semantic web that stores geographical knowledge in a structured form. Based on a geographic knowledge base and a geospatial database, intelligent interactions with virtual geographical environments can be realized by natural language question answering, entity links, and so on. In this paper, a knowledge-enhanced Virtual geographical environments service framework is proposed. We construct a multi-level semantic parsing model and an enhanced GeoKG for structured geographic information data, such as digital maps, 3D virtual scenes, and unstructured information data. Based on the GeoKG, we propose a bilateral LSTM-CRF (long short-term memoryconditional random field) model to achieve natural language question answering for VGEs and conduct experiments on the method. The results prove that the method of intelligent interaction based on the knowledge graph can bridge the distance between people and virtual environments.
\end{abstract}

Keywords: virtual geographical environments (VGEs); knowledge graph (KG); geographic knowledge graph (GeoKG); semantic conversion model; question answering (QA)

\section{Introduction}

Virtual geographical environments (VGEs) were proposed in the late 1990s and have since been deeply studied with regards to visualization, geographic simulation, predictive analysis, knowledge sharing, collaborative modeling [1-6], and so on. With the development of artificial intelligence and big data, intelligent VGEs have to meet the new challenges of geographic knowledge engineering [7]. VGE knowledge engineering provides the supporting theory, method, and technical detail for knowledge-based intelligent VGEs. VGE knowledge involves an evolving process including knowledge generation, integration, and sharing. Intelligent VGEs, based on knowledge, impose higher requirements on the interactivity and intelligent services and will improve the level of intelligence of geographic knowledge services for VGEs.

The core of intelligent VGEs is the formal expression of geographic knowledge. Its purpose is to transform the data, information, and scenes of a virtual geographic environment into "knowledge" that can be recognized by computer, so that computers can understand virtual geographic environments more easily. It can be used in knowledge search, geographic reasoning [8], spatial cognitive simulation $[9,10]$, natural language interaction, and so on.

In terms of the interaction between humans and virtual geographic environments, a lot of work has been done on immersion and interaction by many researchers. Virtual reality, computer graphics, 
and other technologies have enabled better interaction and immersion in VGEs. In addition to using physical devices, such as virtual gloves and helmets, to improve interactivity, question answering is an important natural process. Through natural language interaction, the natural communication between a human and their virtual environment can be realized. Computers must understand the human's intention. Natural language interaction for VGEs requires a knowledge base. It is urgent to address the description, storage, and management of geographic knowledge. The knowledge graph is a good technology to formally represent the knowledge.

In the field of artificial intelligence, technologies such as knowledge graphs (KGs), knowledge representations, and knowledge recommendations have been deeply studied, in order for robots to recognize the real world. They have been widely used in the fields of intelligent search, machine translation, and machine understanding. The core idea of a knowledge graph is to construct a semantic knowledge network which bridges the distance between humans and computers. KGs can be used in VGEs for knowledge generation, representation, and intelligent services.

Research on the knowledge modeling of VGEs based on knowledge graphs is a new topic for intelligent VGEs. It has the following motivations:

(1) Improving the cognitive efficacy of VGEs. Semantic knowledge searches, knowledge recommendations, and intelligent question answering are the main application directions of knowledge graphs. Based on a knowledge graph, natural language interaction with virtual environments can be realized, the immersion and interactivity of VGEs can be further improved, the distance between humans and VGEs can be shortened, and the cognitive effects of VGEs can be improved.

(2) Sharing the knowledge of VGEs. Virtual scenes are represented as scenes, layers, entities, and so on. GeoKG involves extracting the semantic relations between geographic ontologies, concepts, and entities, constructing a semantic relationship network of the VGE objects.

(3) Promoting an intelligent level of knowledge services for VGEs. Based on the database, model library, and knowledge base, the computer can understand human intentions at a natural language level, in order to realize natural language question-and-answer knowledge search and promote intelligent knowledge services.

At present, encyclopedic knowledge graphs have developed rapidly, and many encyclopedic knowledge bases have emerged, which have been successfully used in knowledge search and intelligent question answering. However, the geographic knowledge of an encyclopedic knowledge graph is mostly extracted from encyclopedic websites, whose knowledge sources are limited and not more scientific. The virtual geographic environment is based on a digital map, elevation model, remote sensing image, three-dimensional building, and other multi-source geographic information. The formal expression of virtual geographic environment is essentially a formal representation of the basic geographic information. Therefore, based on Chinese knowledge graph, we extract geographic knowledge from the basic geographic information data of virtual geographic environment- to complete the Chinese encyclopedic knowledge graph, and then use natural language question answering technology to realize intelligent interaction of virtual geographic environment scenes.

Our work achieves a method of intelligent question answering interactions with VGEs based on GeoKG. The main contributions of this study can be summarized as follows:

(1) A knowledge-enhanced service framework and multi-level semantic parsing model for VGEs is proposed. The formal expression of virtual geographic environment knowledge based on geographic data is realized, and the geographic knowledge base is merged with an encyclopedic knowledge graph.

(2) A bilateral LSTM-CRF (long short-term memory-conditional random field) model and multi-feature logistic model for intelligent interaction is proposed, and

(3) The applicability of the proposed method in two practical scenarios-geo-entity research and question answering interactions-based on GeoKG is demonstrated. 


\section{Related Work}

The core idea of VGE knowledge searches based on GeoKG is to construct a semantic knowledge network. The framework is shown in Figure 1. A geographic knowledge base is a semantic web-in comparison, a geographic information database is based on a geo-spatial model such as a vector model, a raster model, or a volume model. Using a multi-level semantic conversion model, a geographic information database can be enhanced to a geographic knowledge base in order to enhance the knowledge service.

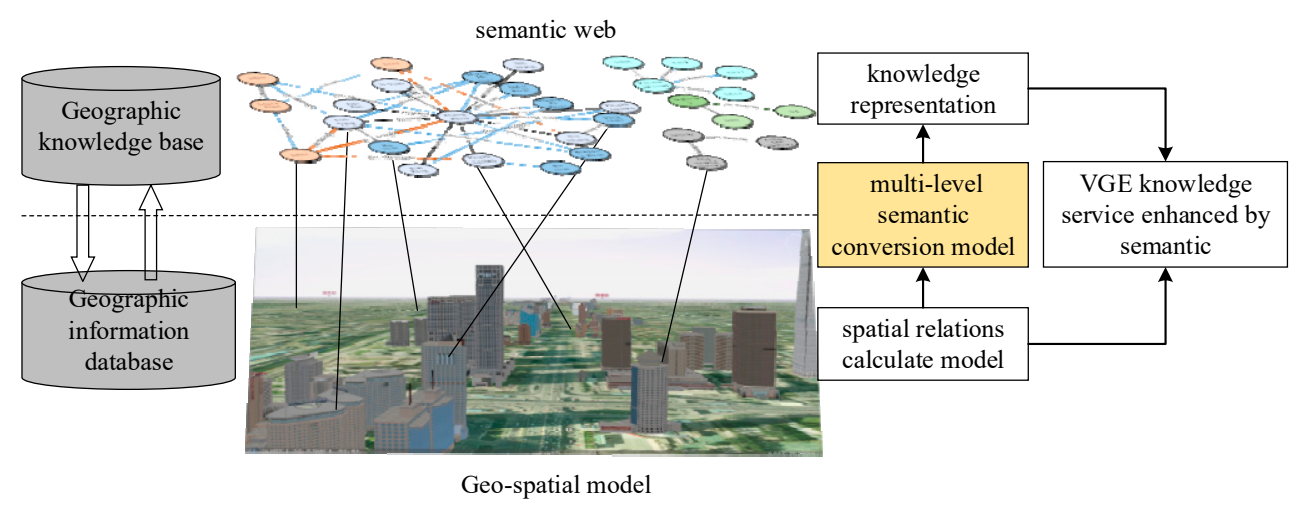

Figure 1. Framework of VGE service enhanced by geographic knowledge.

\subsection{Knowledge Representation of VGE}

Geographic knowledge is the higher level of geographic information. The expression and sharing of geographic knowledge are important in intelligent virtual geographic environments (VGEs). Mehdi Mekni [8] defined the notion of environment knowledge (EK) as a description (like a formal specification of a program) of the spatial concepts (geographic features) and relationships (topologic, semantic) that may exist in a geo-graphic environment. Lin [11] divided geographic knowledge into three levels: factual knowledge, rules and control knowledge, and decision-oriented knowledge. He proposed that the knowledge in VGEs (VGE-K) is geo-information related to various geoscience questions, the placement of phenomena in a geo-context and the extraction of geospatial rules [7]. Robert Laurini $[12,13]$ presented a tentative conceptual framework for managing practical geographic knowledge, and geographic knowledge base (GKB) includes geographic objects, geographic structures, geographic relations, geographic rules, geographic ontology, gazetteer, physico-mathematical models, and external knowledge.

For knowledge representation and reasoning in multiagent geo-simulations, Mehdi Mekni proposed the informed virtual geographic environments (a semantically enriched and geometrically accurate VGE) [14]. He used the GIS data to generate an informed topologic graph (ITG), where each node corresponds to the map's triangles, and each arc corresponds to the adjacency relations between these triangles [15]. To achieve the intelligent VGEs, Lin [11] presented the knowledge engineering of VGEs (VGE-KE) which is a promising means of providing geo-knowledge.

From the perspective of geographical knowledge representation, we divide geographical knowledge into factual knowledge and process knowledge. Factual knowledge mainly refers to the knowledge (so-called "lightweight" knowledge) that reflects the external characteristics and connections of geographical entities, such as geographical terms, gazetteer, geographical distribution and geographical data. Procedural knowledge refers to the geographical model describing the spatial-temporal transformation of geography, such as the law of geographical evolution and the law of geographical prediction, which belongs to the knowledge with strong specialty. 


\subsection{Knowledge Graph}

The knowledge graph is a branch of artificial intelligence which has been widely studied. The knowledge graph (KG) was formally proposed in 2012 by Google to achieve a more intelligent search engine. A KG is essentially a knowledge base called a semantic network, and is a knowledge base with a directed graph structure, in which the nodes of the graph represent entities or concepts, and the edges of the graph represent various semantic relationships between entities (or concepts) [16].

Knowledge graphs are characterized by large scales, rich semantics, high quality, and friendly structures. They are mainly used in knowledge searches, intelligent question answering, and to assist decision-making. A knowledge graph is very suitable for representing factual knowledge in a virtual geographic environment.

According to its service objects, a knowledge graph can be divided into an open knowledge graph and a domain knowledge graph. A large number of knowledge graphs have been produced [17]. Examples of open knowledge graphs are WordNet, Freebase [18], DBpedia [19], YAGO [20], and Zhishi.me [21], as well as the Chinese CN-DBpedia [22]. Examples of domain knowledge graphs are KnowItAll [23], TextRunner [24], and Nell [25], among others. Although the linked open data cloud contains some multilingual knowledge graphs, most of the knowledge (including concepts, entities, triples, etc.) is still denoted in English, and the number of Chinese knowledge graphs is quite few [26].

The encyclopedia knowledge graph mainly extracts knowledge from encyclopedia websites and lacks geographic knowledge. To meet the needs of this study, a geographic knowledge graph (GeoKG) needs to be generated by extracting geographic information from the virtual geographic environment.

\subsection{Geographic Knowledge Graph}

A geographic knowledge graph (GeoKG) is an expanded knowledge graph with information of geography. It is a structured geo-semantic knowledge base. It organizes data in form of geographic entities and describes geographic concepts and entities in the form of "entity-relationship-entity" triples, as shown in Figure 2.

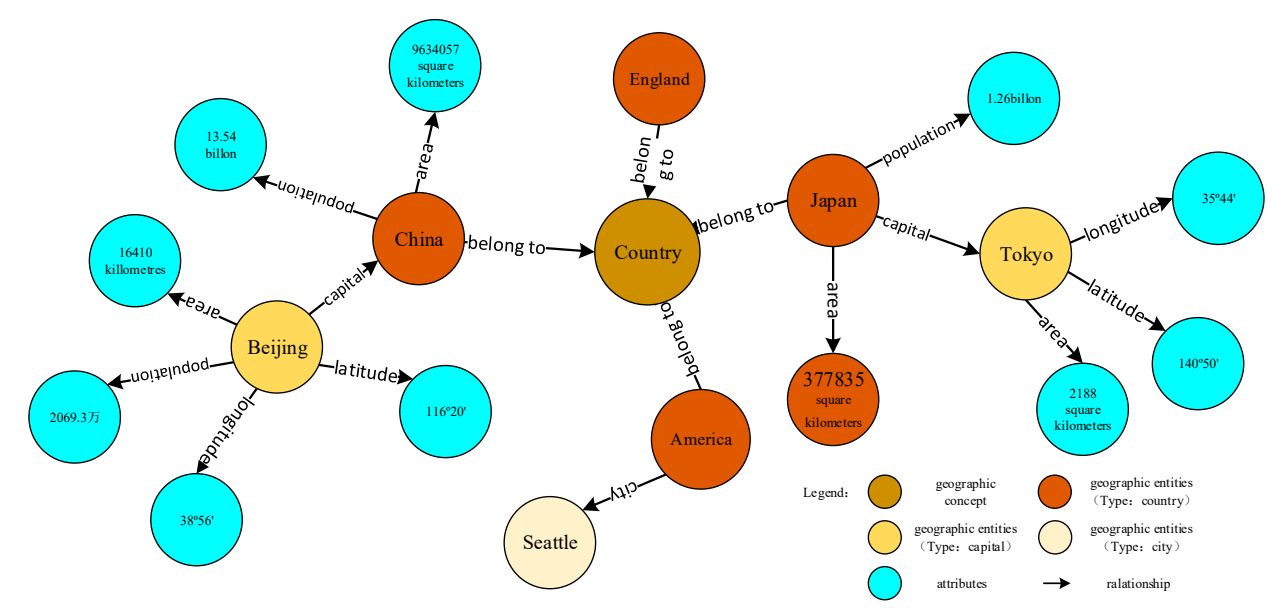

Figure 2. Example of a geographic knowledge graph.

A geographic entity refers to a geographic phenomenon that cannot be divided into similar phenomena, according to a certain standard in the geospatial world. It has a time, space, geo-relationship, and other various attributes.

Geographic knowledge is described in RDF (resource description framework) triples, and the form of visualization is a "point-edge" graph, as shown in Figure 2. The points represent geographical concepts, entities, and attribute values. Edges represent the various relationships, including relationships between concepts and concepts, concepts and entities, entities and entities, entities and attributes, and attributes and attribute values. 
For example, as shown in Figure 2, $<$ China, belong to, country $>$ is a relationship between entity and concept, $<$ China, capital, Beijing $>$ is a relationship between entity and entity, and $<$ Beijing, population, 20,693,000 > is a relationship between entity and attribute.

A GeoKG is constructed using a "top-down" approach, which is mainly divided into a schema layer and data layer, as shown in Figure 3. The schema layer is mainly a geographic ontology or concept layer, while the data layer is mainly geographic entities and related attributes. The basic idea is to realize the construction of geographic ontologies and concept layers, according to geographic ontologies, based on geographic information data (such as place name data, topographic maps, and so on), to achieve the extraction of geographical entities, related attributes, combined with the filling of relationships by thematic websites and encyclopedia data entities.

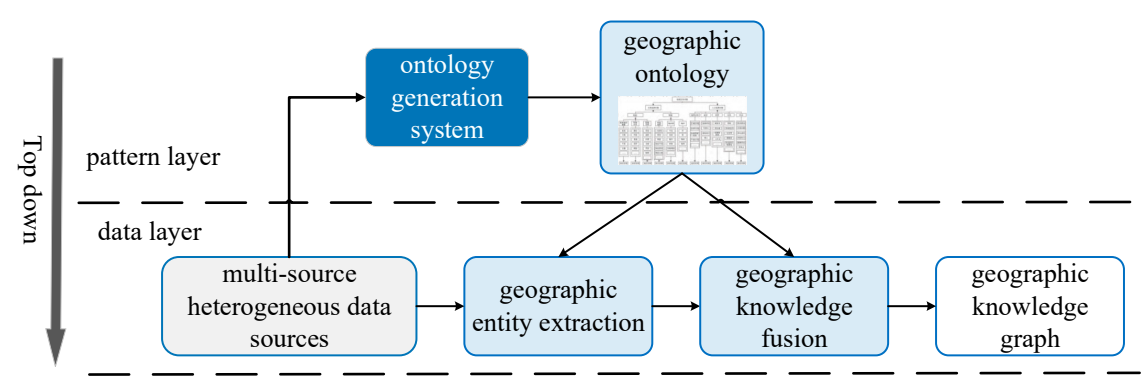

Figure 3. The framework of geographic knowledge graph construction.

There already exist some GeoKG; for example, the GeoNames Ontology, OSM Semantic Network [27], LinkedGeoData, GeoWorldNet, and so on [28]. Research on the construction of GeoKGs mainly includes geographic entity extraction [29-31], topological and azimuthal relation extraction [32,33], and KG storage [34-36]. In terms of applications, there are the typical geo-semantic semantic sharing network systems (Geo-Wiki) [37] and the geographical knowledge-based dictionary (KIDGS) [38].

A knowledge graph for a VGE is mainly extracted from different VGE scenarios. For heterogeneous structured data such as maps, a semantic conversion model must be established, and geographic knowledge is acquired through geographic entity extraction, de-duplication, and alignment in order to complete and enhance the KG.

\subsection{Question Answering Based on Knowledge Graph}

Knowledge-based question answering methods can be divided into two categories: semantic parsing-based (SP-based) and information retrieval-based (IR-based) methods. Due to the emergence of large knowledge bases, information retrieval methods are popular. IR-based methods firstly obtain a series of candidates from a knowledge base using a relatively rough method, performs feature extraction on the questions and candidates, uses these to sort the candidates, and eventually selects the highest score as the final answer.

With the development of deep learning, a series of natural language question answering methods based on representation learning has emerged. Representation learning is mainly directed at the representation of semantic relations [39]. It is mainly divided into two types: mapping matrix or mapping vector. Mapping matrix methods mainly include structured models [40], semantic matching energy (SME) models [41], latent factor (LF) models [42], single layer (SL) models [43], and so on. Mapping vector methods mainly include TransE models [44], TransH models [45], TransR models [46], and TransD models [47], among others. An end-to-end approach based on learning natural language questions and answers is used to solve the question and answer problem, omitting the intermediate steps, by completely mapping the text to the knowledge time mapping from the data, lacking a semantic analysis of the displayed questions. The accuracy of these methods is often insufficient. 
Entity linking is one of the key technologies for the interactive search of VGE scenes based on a knowledge graph. In-depth research has been conducted in the field of knowledge graphs. The representative methods are: (1) probabilistic generation model-based methods: building a probabilistic model between candidate entities and entity referential terms [48], improving the efficiency of entity linking, (2) thematic model-based methods: training latent Dirichlet allocation (LDA) topic models with training datasets, realizing entity elimination through semantic similarity [49], (3) graph-based methods: building a graph-based model to achieve target entity selection [50], and (4) deep neural network model-based methods: training entity representation models in a supervised or semi-supervised manner using deep neural network models, sorting them according to semantic similarities [51].

In summary, knowledge search in VGEs is mostly based on keywords-there is no geographic knowledge base as support. It is difficult to understand the intentions of people and achieve effective question-and-answer knowledge searches. Note that our goal is to use semantic parsing models to realize knowledge enhancement and complement, and to implement natural language question answering interactions and knowledge searches.

\section{Multi-Level Semantic Parsing Model for VGE}

In extracting geographic knowledge from a VGE scene and realizing the transformation of "view (graphic) $\rightarrow$ semantic", the core idea is to establish an interpretative model mapping from scene to semantics, as shown in Figure 4. The multi-level semantic parsing model is used to resolve the topological, directional, and distance relations in semantic descriptions at different scales of the virtual scene.

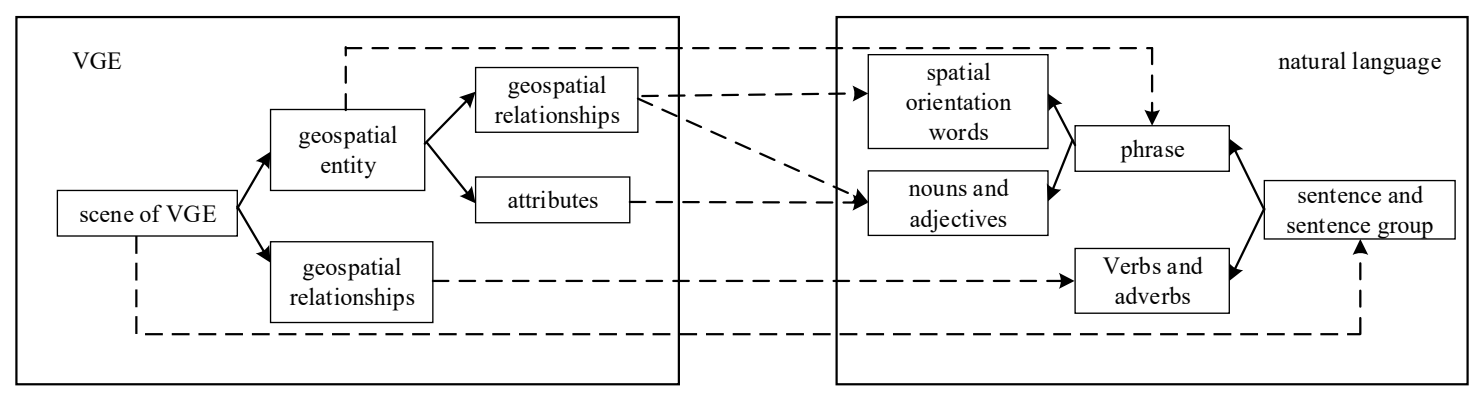

Figure 4. Feature mapping between VGE and natural language.

\subsection{Resolving Spatial Topological Relations}

The model mainly considers the types of geographic entities and establishes a mapping of spatial topological relations expressions and spatial description vocabularies (as shown in Table 1). As shown in Figure 5, based on vector data (usually for small or medium-scale scenes) and 3D models (usually for large-scale scenes), geo-entities are extracted and, through a geometric calculation function, a spatial topology between the geo-entities is calculated. Then, topological relations and spatial type mapping vocabulary (as shown in Table 2) are used to convert the spatial topology to semantic relations.

Table 1. Mapping between the topological relation vocabulary and a geometric calculation function.

\begin{tabular}{cccccc}
\hline Vocabulary & $\begin{array}{c}\text { Feature A } \\
\text { Type }\end{array}$ & $\begin{array}{c}\text { Feature B } \\
\text { Type }\end{array}$ & $\begin{array}{c}\text { Geometry } \\
\text { Type }\end{array}$ & $\begin{array}{c}\text { Geometric Calculation } \\
\text { Function }\end{array}$ & Sketch \\
\hline \multirow{2}{*}{ flow in } & river & inhabitant & line-polygon & $\left\{\right.$ Line, $\left[\begin{array}{lll}0 & 0 & 1 \\
0 & 0 & 1 \\
1 & 1 & 1\end{array}\right]$, Polygon $\}$ & \\
\hline
\end{tabular}




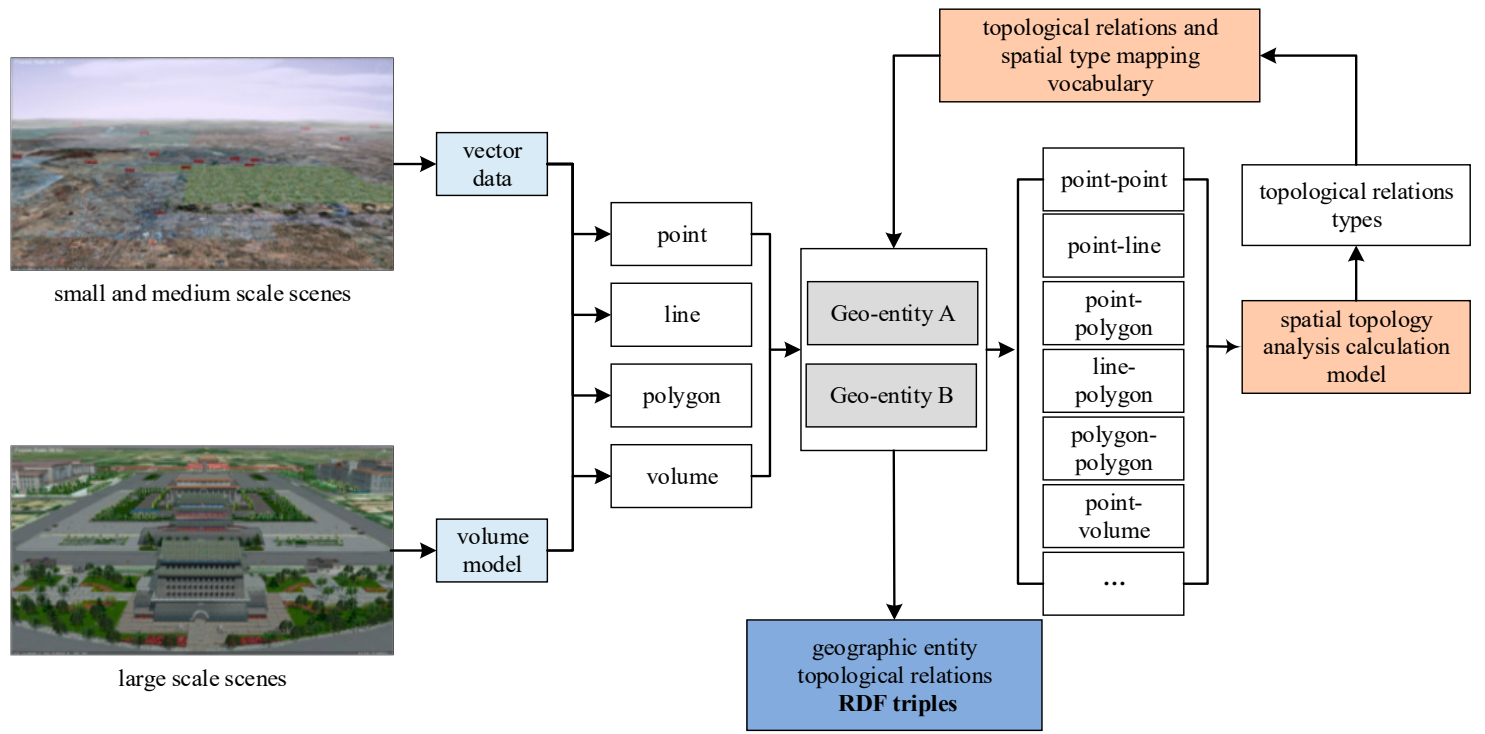

Figure 5. Semantic mapping flow of topological relationships.

Table 2. Dictionary of topological relations mapping to spatial relations.

\begin{tabular}{|c|c|c|c|}
\hline Feature A Type & Feature B Type & Spatial Topological Type & Semantic Relations Vocabulary \\
\hline \multirow{3}{*}{ river } & \multirow{3}{*}{ city } & Included (IN) & $\begin{array}{l}\text { contain, include, belong to, branch, } \\
\text { offshoot, intersection, join, inflow, ... }\end{array}$ \\
\hline & & Externally Connected (EC) & $\begin{array}{l}\text { flow through, flow in, connect, } \\
\text { birthland, join into, ... }\end{array}$ \\
\hline & & Disconnected (DC) & disjoint, be apart, ... \\
\hline building & road & Disconnected (DC) & $\begin{array}{l}\text { besides, adjacent, neighbor, go across, } \\
\text { flow through, overlap, cross, ... }\end{array}$ \\
\hline \multirow{2}{*}{ road } & \multirow{2}{*}{ city } & Externally Connected (EC) & start with, from, begin with, end, ... \\
\hline & & Disconnected (DC) & go across, pass through, .... \\
\hline
\end{tabular}

For example, the description "river flowing through China" expresses the topological relationship of "line-area", is identified as "intersecting" type through topological calculations, and is mapped into the description of language relations, such as "flow through" or "flow-in". Other examples of resolving spatial topological relations are shown in Table 3.

Table 3. Examples of resolving spatial topological relations.

\begin{tabular}{ccc}
\hline Scene of VGE & $\begin{array}{c}\text { Spatial Topological } \\
\text { Representation }\end{array}$ & Semantic Graph Representation \\
\hline
\end{tabular}


Table 3. Cont.

Scene of VGE

\subsection{Resolving Spatial Orientation Relations}

The semantic analysis of orientation relationships needs to consider the constraints of topological and distance relations. As shown in Figure 6, we first need to describe the types of geometric features (such as point, line, surface, and volume) and separately calculate the spatial orientation, eight-direction spatial azimuth model for small-scale scenes, and three-dimensional azimuth model for large-scale scenes. The former divides a two-dimensional plane into eight parts, as shown in Figure 7 (left); the latter divides three-dimensional space into 27 parts, as shown in Figure 7 (right). Then, according to the mapping vocabulary, the semantic relations between entity A and entity B are confirmed.

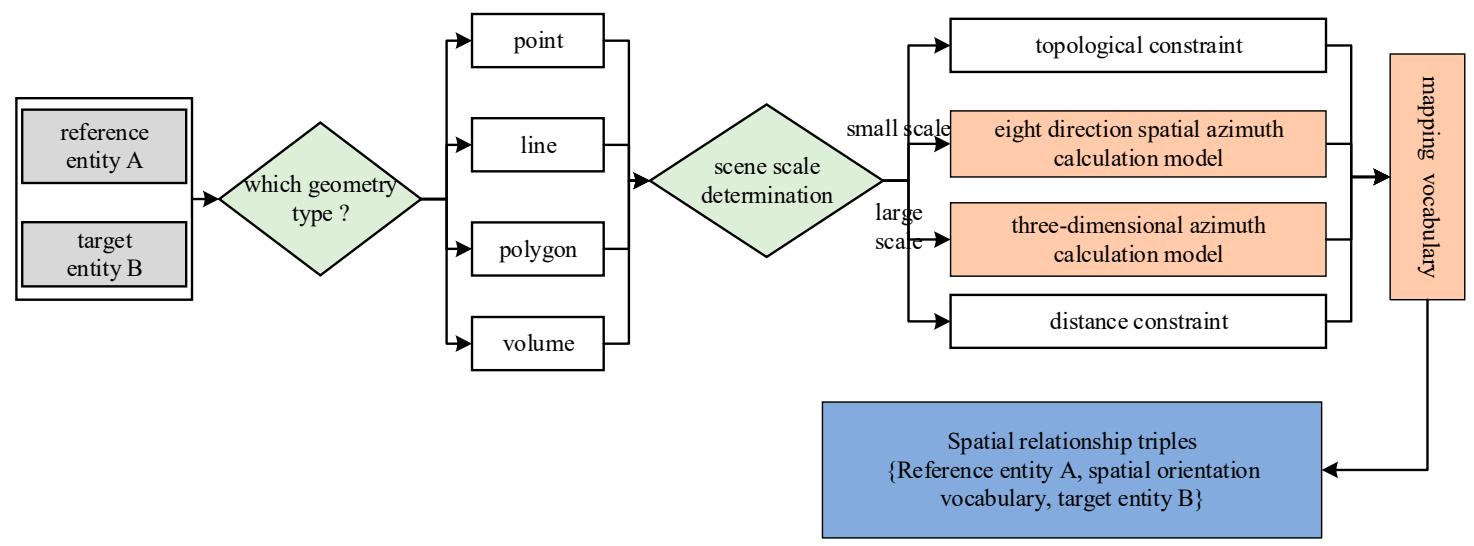

Figure 6. Semantic conversion model of orientation relations.
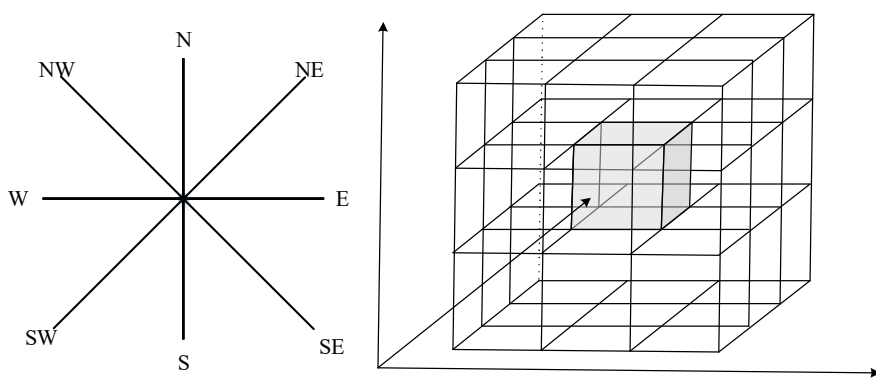

Figure 7. Eight (left) and 27 (right) parts centered on the reference entity, for the two- and three-dimensional cases, respectively. 
For example, the expression corresponding to a "southeast" orientation description vocabulary is shown in Equation (1). When the spatial orientation relation between $A$ and $B$ satisfies Equation (1), the defined target object $A$ (dot) is located a southeast direction from the reference object $B$ (dot). In Equation (1), $S R$ denotes the azimuth relation expression of the target $A(\mathrm{dot})$ and reference $B$ (dot), $D I R$ denotes a directional relationship function, TOP denotes a topological relationship category, and DIS denotes a spatial distance. The example of resolving spatial orientation relations is shown in Table 4.

$$
S R(A, S E, B)=\left\{D I R=\left(\frac{13 \pi}{8}<\theta<\frac{15 \pi}{8}\right), T O P=\left[\begin{array}{lll}
0 & 0 & 1 \\
0 & 0 & 1 \\
1 & 1 & 1
\end{array}\right], D I S>0 m\right\} .
$$

Table 4. Examples of resolving spatial orientation relations.

Scene of VGE

\subsection{Resolving Spatial Distance Relations}

The semantic conversion model of distance relations mainly uses distance calculations in twoor three-dimensional space to determine semantic relations with natural language descriptions. The corresponding distance $\left(D_{i j}\right)$ is shown in Equation (2). Then, distance relation type and semantic relations mapping vocabulary (as shown in Table 5) are used to convert the spatial distance to semantic relations.

$$
D_{i j}=\sqrt{\left(x_{i}-x_{j}\right)^{2}+\left(y_{i}-y_{j}\right)^{2}+\left(z_{i}-z_{j}\right)^{2}} .
$$

Table 5. Vocabulary of distance relations mapping to spatial relations semantic description.

\begin{tabular}{cc}
\hline Distance Relation Type & Distance Relational Semantic Vocabulary \\
\hline $\begin{array}{c}\text { Distance between point, line, polygon, and volume } \\
\text { elements }\end{array}$ & far, far away, farther, near, nearer, close, nearby, \\
around, distant, remote, ...
\end{tabular}

Qualitative expression needs to be associated with qualitative distances under certain distance scale standards. Thresholds were considered with spatial scales in order to achieve conversion from qualitative to quantitative distances. A threshold set is defined as $D\left\{D_{i}, D_{i+1}, D_{i+2}, \cdots\right\} \subseteq D$. Due to different scales, in practice, the description of the same qualitative distance may correspond to totally different quantitative distances. For example (as shown in Table 6), in China, "Tiananmen Square" is $10 \mathrm{~km}$ away from "Olympic Park" and it is generally qualitatively expressed that they are "far"; whereas "Beijing" is about $120 \mathrm{~km}$ away from "Tianjin", but the two are typically qualitatively expressed as "near". 
Table 6. Examples of resolving spatial distance relations.

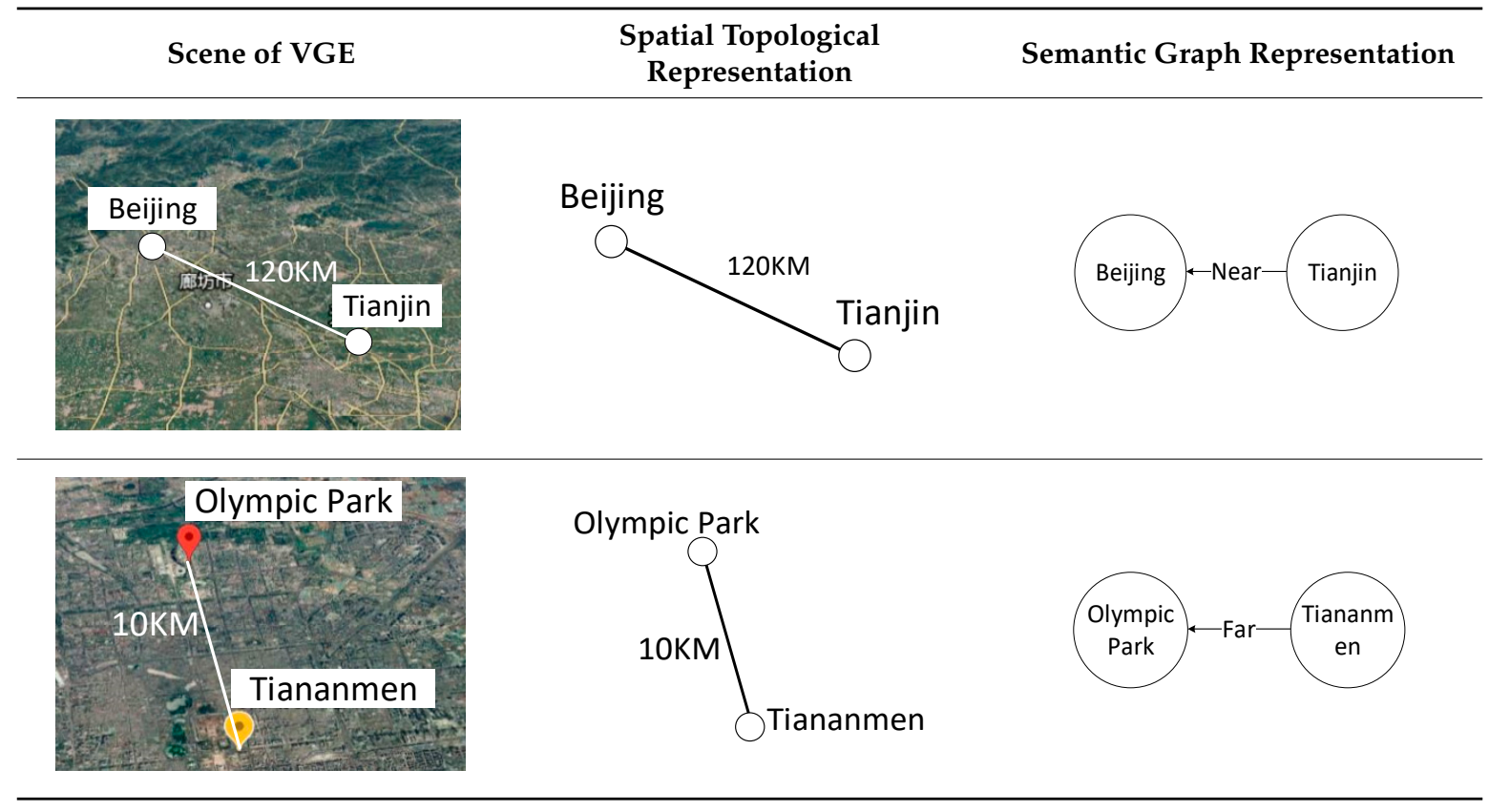

\section{Multi-Element Geographic Knowledge Extraction Model}

VGE data are characterized by heterogeneity and coming from multiple sources. Structured geographic information data includes two-dimensional vector data, three-dimensional model data, and so on. Basic geographic information data is a symbolic understanding of the real world. It is characterized by rich knowledge and accurate spatial relationships. Thus, it is an important source for GeoKG.

The key idea of the multi-geographic geographic knowledge extraction model is to design different models for a three-dimensional scene. The geographic knowledge extraction model includes: (1) extraction of geographic entities and spatial relationships, and (2), based on the multi-level semantic analysis model, and according to the varying characteristics of different feature layers, construction of a map from spatial relational data to RDF (resource description frameworks) semantic triples.

The process of knowledge extraction from VGE structured data is illustrated in Figure 8. According to the structure of data at different element levels stored in the relational database, the extraction model of geographic entities and semantic relations is designed and represented as geographical knowledge. The triples are stored in the graph database.

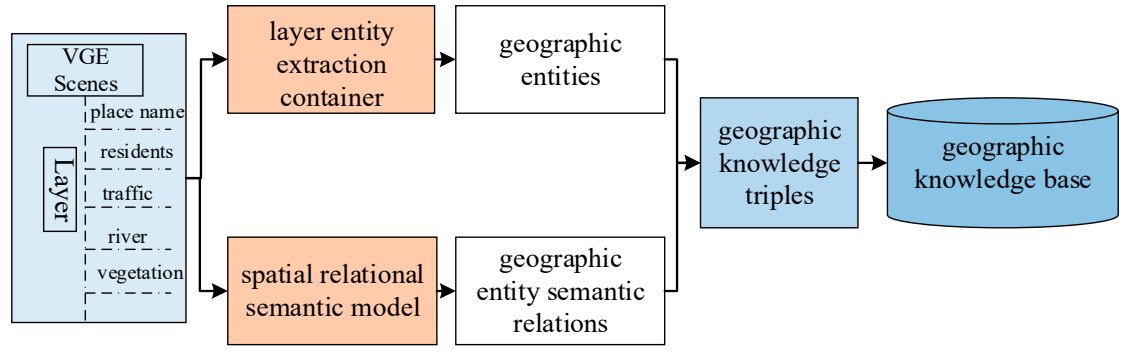

Figure 8. Geographic knowledge modeling based on vector data.

The main method used is D2R structured data extraction. D2R (relation database to RDF) refers to converting the structured data in a relational database management system (RDBMS) into semantic RDF resources. We aim to establish a specific map based on the ontological model and to realize the mapping of relational data to RDF semantic data. The flow is demonstrated as followed: 
Firstly, for the basic format of the structured data stored in different data layers, determine the extracted content, and establish the extraction model for different data layers;

Secondly, mapping specification based on the ontological model is constructed to associate the structured data with concepts and entities in the knowledge graph.

Finally, the concepts, entities, and attributes of association mapping are stored as triples.

Taking a 1:250,000 map as an example, geographic semantic knowledge extracted from the relational database (schematic diagram) is demonstrated in Table 7.

Table 7. Semantic relationships of geographic entities extracted from a 1:250000 map of Zhengzhou (in China).

(a) original 2D map of VGE

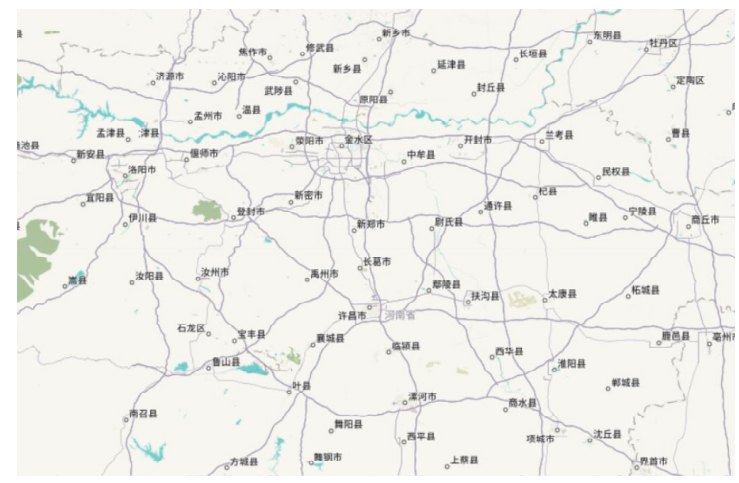

(b) topological map

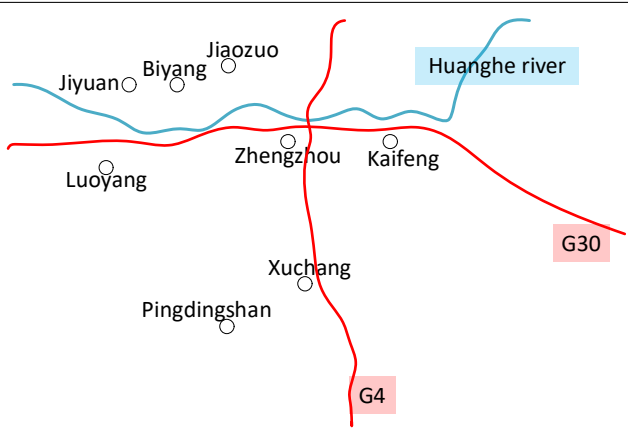

(c) multi-element geographic knowledge graph
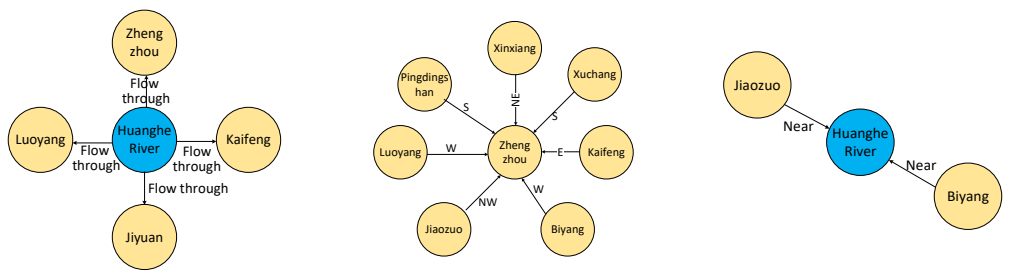

(d)merge the geographic knowledge into one graph

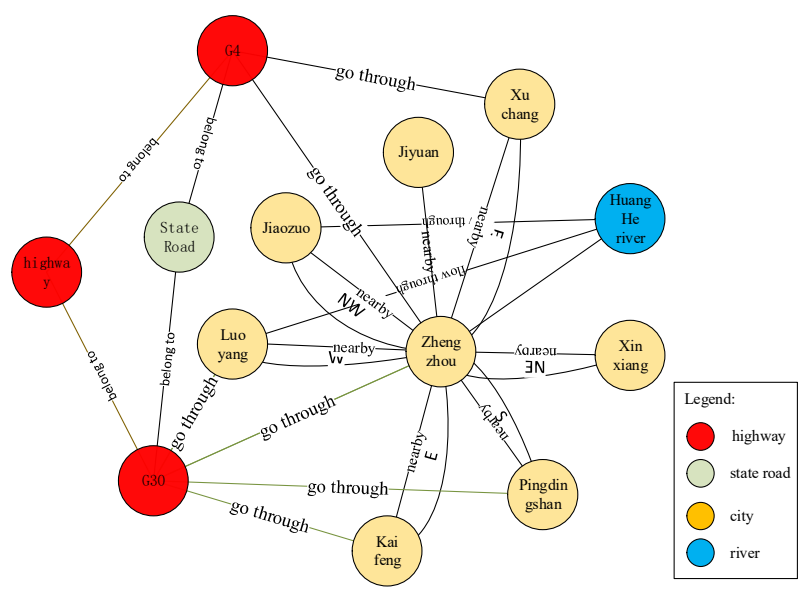




\section{Intelligent Interaction with VGE Based on Geographic Knowledge Graph}

Due to the complexity of multilingual relations in natural language question-answering (such as "what are the cities near the east of Zhengzhou?"), we only consider unilateral questions (e.g., "What are the cities near Zhengzhou?"). As shown in Figure 9, the key technologies include: geographic entity identification, resource mapping, and querying statement generation.

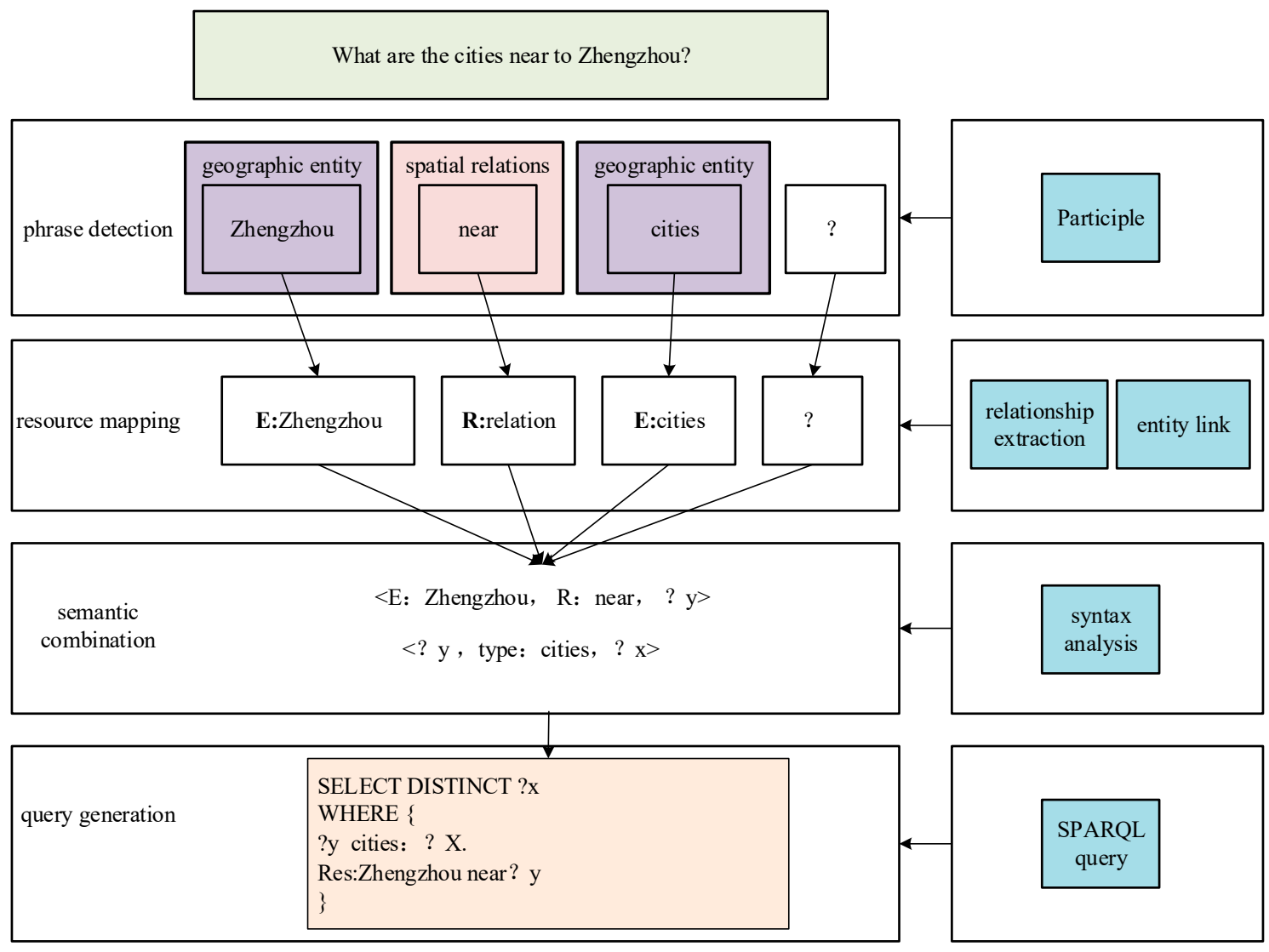

Figure 9. Question answering flow chat.

We use a semantic parsing-based (SP-based) approach to achieve natural language question and answer queries. This can be divided into: (1) identification of geographic entities, which identifies the geographic entity from the natural language text, and (2) geographic entity linking, in which the identified geographic entities are mapped to the geographic knowledge base in order to obtain candidate entities.

\subsection{Bilateral LSTM-CRF Model for Geographic Entity Identification}

A LSTM (long short-term memory) model is characterized by its ability to preserve contextual information. A CRF (conditional random field) model can take into account the influence between front and back annotations from the sentence level. Combining the advantages of the two models, we used a bilateral LSTM-CRF [52] model to improve the accuracy of geographic entity identification in question answering.

A LSTM model is implemented by calculating the logarithmic probability of the entire question (see Equation (3)), which, in order to meet the natural language grammatical structure, can obtain higher probability.

$$
L P(S)=-\sum_{i=1}^{n} \log P\left(W_{i}\right)
$$


where $\boldsymbol{P}\left(\boldsymbol{W}_{\boldsymbol{i}}\right)$ represents the predicted probability of the $\boldsymbol{i}$-th word in the question. A smaller $\boldsymbol{L P}(\boldsymbol{S})$ implies a higher probability that the statement $S$ conforms to the grammar. The logarithmic probability obtained by a basic LSTM model only considers the position and contextual information of candidate entities and does not use any information of the candidate entities itself. We extracted two additional features to improve the LSTM model: the length $L$ (in words) of the candidate geographic entity and the IDF (inverse document frequency) value of the geographic entity.

One of the CRF linear layers is added after the hidden layer of the bilateral LSTM network. The model structure is shown in Figure 10. By introducing a state transition matrix $A$ and setting the matrix $P$ to be the output sequence of the annotation sequence $y=\left(y_{1}, y_{2}, \cdots, y_{n}\right)$ corresponding to the output observation sequence $X$ of the double-layer LSTM network, the predicted output is:

$$
\mathrm{s}(X, \mathrm{y})=\sum_{i=1}^{n}\left(A_{y_{i}, y_{i+1}}+P_{i, y_{i}}\right),
$$

where $A_{i, j}$ represents the probability of transition from the $i$-th state to the $j$-th state in the time series and $P_{i, j}$ represents the probability that the $i$-th word in the input observation sequence is the $j$-th label.

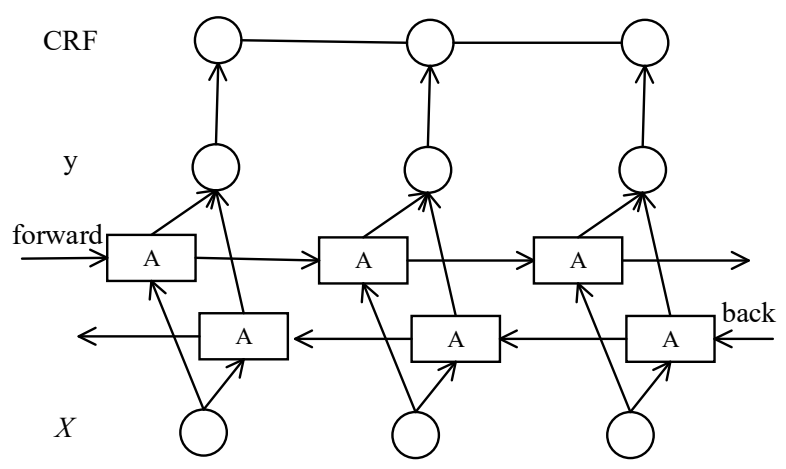

Figure 10. Bilateral LSTM-CRF model structure.

\subsection{Multi-feature Logistic Model for Geographic Entity Linking}

Geographic entity linking is used to calculate the semantic similarities between candidate geographic entities and geographic referential entities. We apply multiple characteristics to implement the logistic regression model. In order to enable multiple feature values, to better represent the semantic similarity of entities, after completing entity identification and matching the set of candidate entities, it is necessary to calculate the feature values for all entity pairs to evaluate the linking possibility between them, as is shown in Figure 11.

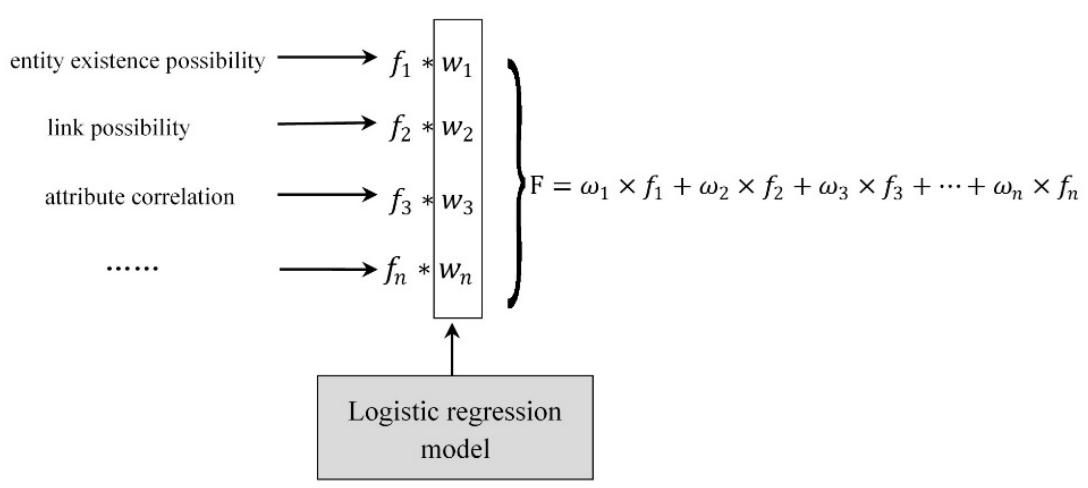

Figure 11. Weighted calculation of eigenvalues. 
The weighted eigenvalue $\boldsymbol{F}$ between the entity $\boldsymbol{m}$ and the candidate entity $\boldsymbol{e} \in \boldsymbol{E}_{\boldsymbol{m}}$ can be calculated, as follows:

$$
F=\omega_{1} \times f_{1}(m, e)+\omega_{2} \times f_{2}(m, e)+\omega_{3} \times f_{3}(m, e)+\ldots+\omega_{n} \times f_{n}(m, e) .
$$

In the matching candidate entity set, the entity with the highest weighted feature value will become the final matching pair of entity $m$.

The specific proposed method is to select the geographic knowledge base as a training data set and extract the entity set $L=\left\{\left\langle m_{i}, e_{i}\right\rangle\right\}$ whose linking information is sufficient (that is, there is a large number of linkages) as the training data, and then use the logistic regression model to calculate the weight of each feature value. For an entity $m$ and corresponding candidate entity $e$, each eigenvalue weight $\omega$ must satisfy the following relationships:

$$
\omega \cdot\left(f\left(m, e^{*}\right)-f(m, e)\right)>0,\left(e \in E_{m}, e \neq e^{*}\right)
$$

where $\omega=\left\langle\omega_{1}, \omega_{2}, \omega_{3}, \ldots, \omega_{n}\right\rangle, f=\left\langle f_{1}, f_{2}, f_{3}, \ldots, f_{n}\right\rangle$. The probability that the entities $e_{1}$ and $e_{2}$ are linked to the entity $m$ can be calculated using the sigmoid function, where the calculation formula is as follows:

$$
P\left(\left(e_{1}>e_{2}\right)=\text { true }\right)=\frac{1}{1+e^{-\omega\left(f\left(m, e_{1}\right)-f\left(m, e_{2}\right)\right)}} .
$$

If $s\left(m, e_{1}\right)>s\left(m, e_{2}\right)$, then $P\left(\left(e_{1}>e_{2}\right)=\right.$ true $)>0.5$; if not, then $P\left(\left(e_{1}>e_{2}\right)=\right.$ true $)<0.5$. The final weights can be determined by maximum likelihood estimation and Logistic regression models. Then, the weight $\omega$ is substituted into formula 5 to solve the weighted eigenvalues, in order to discover and predict new entity links.

\section{Discussion}

In this section, the case study of formalizing VGE scene of Beijing was constructed by using multi-feature logistic model, and the experiment was designed for testing the interaction with VGE by natural language question answering. Then, the results were analyzed to evaluate the ability of this method for intelligent interaction with a virtual geographic environment based on a geographic knowledge graph.

The experimental data mainly included three parts: basic geographic information data of the VGE, corpus data, and encyclopedia knowledge base data. The basic geographic information data of the VGE included two-dimensional vector data and three-dimensional model data of Beijing city in China. The encyclopedia knowledge graph is CN-DBpedia, which has 80 million entities, and 120 million relations.

The experimental platform was a Windows 10 system, the database was MongoDB, and the development environment used was Eclipse. The results of knowledge card are shown in Figure 12. The knowledge card includes: relationships of Entities (shown as a "node-edge" graph), summary of entity, infobox of entity, and geographic entity category.

Using the knowledge graph, in combination with three-dimensional scenes, question and answer (Q\&A)-based VGE interactions were realized. The results are shown in Figure 13. The system supports two types of interaction:

(1) Knowledge graph-based interaction. If an entity node is clicked in the knowledge graph visualization view, while the node has a geographic location attribute, the corresponding view on the right side of the map will dynamically jump to the corresponding geographic location. For example, if the entity node with label "Shanghai" (in Chinese) is clicked in the Knowledge Graph, the corresponding virtual scene viewpoint will dynamically jump to Shanghai.

(2) Interaction by Q\&A. If "where is the capital of China" is typed into the search box, the corresponding map will retrieve the entity "Beijing" and its related attribute information will be displayed in the lower left view. The map view on the right side will follow the linkage and automatically roam the global virtual geographic environment to the Beijing area. 


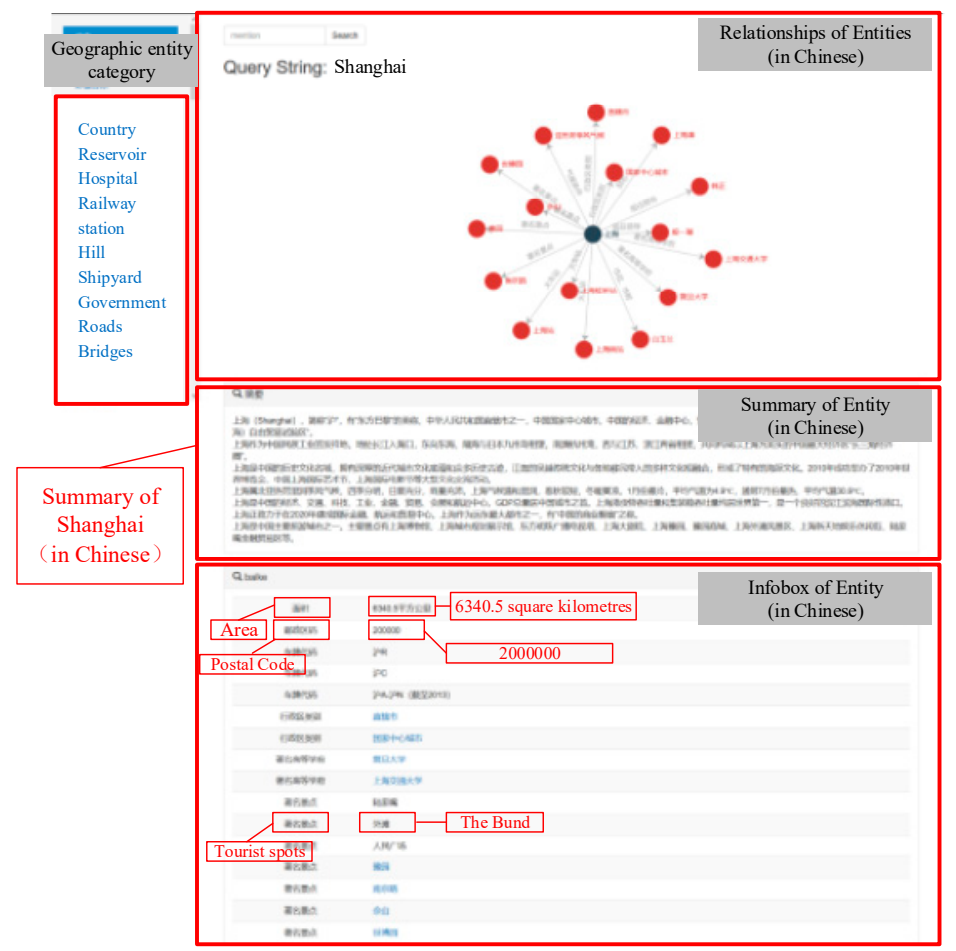

Figure 12. The example of Chinese geographic knowledge card.

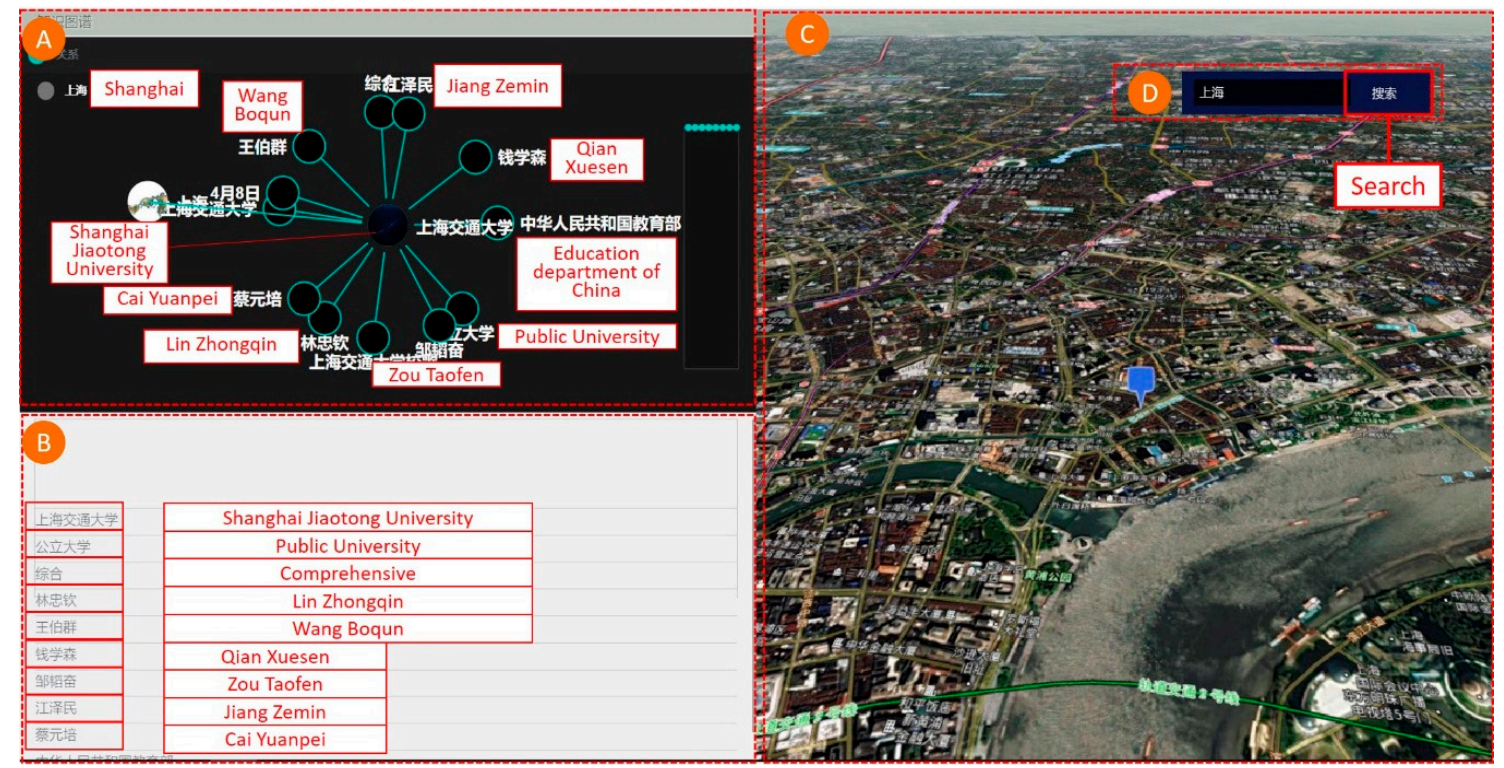

Figure 13. Q\&A based interaction with VGE ((A): Chinese geographic knowledge graph; (B): geographic entity information; (C): 3D VGE scene; and (D): Q\&A search bar.).

In summary, users can interaction with VGE using the human-computer conversation on the natural language. The method of intelligent interaction based on GeoKG can bridge the distance between people and virtual environments. However, there are still many deficiencies in our work, and we need to make further improvements in the following areas:

(1) Semantic transformation of large-scale scene models (such as BIM (building information modeling) models) to satisfy the semantic search of and interaction with interior spaces;

(2) Semantic search and questioning in larger geographic fields. We only used geographic data of the Beijing urban area in our experiment. For a large-scale knowledge graph, the data volume was too 
small. It is necessary to use global crowdsourcing techniques to achieve the semantic extraction and transformation of massive data for global geospatial information.

(3) Natural language question answering needs to be strengthened through geographic language training. The training data used in this study used a common corpus; therefore, a special corpus in the field of geography needs to be constructed for geo-entity recognition and phrase segmentation, in order to train Q\&A models and to improve the accuracy of geographic question answering.

(4) Further improvements need to be made on the accuracy of natural language question answering. We only used the relevant models to implement Q\&A and did not perform relevant experimental analysis for different corpora and different training models. The next step requires a detailed analysis of the accuracy of the training of the model, in conjunction with a specific geo-corpus to further improve the accuracy of entity links and scene interactions.

In a word, the intelligent interaction of virtual geographic environments could be the research hotspot in this field, and this could be a focus of continuous further research on the formalized representation model, intelligent service, and applications based on the GeoKG in the future.

\section{Conclusions}

VGE knowledge engineering is currently in its infancy. At present, there are few Chinese geographical knowledge graphs, and there is a lack of geographical knowledge in the encyclopedic knowledge graph. In this paper, based on the geographic information data of a virtual geographic environment, geographic knowledge is extracted, and virtual geographic environment scenes are formally expressed as spatial knowledge that can be recognized by computer. The scene interaction of natural language question-and-answer and the virtual geographic environment is realized by using natural language processing technology.

Specifically, with the aim to develop intelligent services for VGE, we constructed the "spatial-to-semantic" conversion model, proposed a method for extracting geographic knowledge from structured geospatial information, realized the enhancement of Chinese geographic knowledge, and proposed a bilateral LSTM-CRF model and multiple feature-influenced logistic model to implement intelligent interactions with a VGE.

Using geospatial information data, language data, and open knowledge base data, experiments were conducted to build a large-scale VGE knowledge graph. Semantic querying of geographic knowledge can be implemented, and related attribute information of entities can be displayed in the form of knowledge cards. Location information is associated with geo-entities. Furthermore, observation viewpoints of virtual scenes can change with Q\&A interaction. This provides a new means for intelligent interaction with VGEs.

Author Contributions: Conceptualization, Bingchuan Jiang; Methodology, Bingchuan Jiang and Liheng Tan; Resources, Yan Ren; Software, Feng Li; Writing_original draft, Bingchuan Jiang; Writing—review \& editing, Bingchuan Jiang, Liheng Tan, Yan Ren and Feng Li.

Funding: This research was funded by the National Natural Science Foundation of China under Grant 41801319, the Science and Technology Program of Henan in China under Grant 182102210147, and the Foundation for State Key Laboratory of Geo-information Engineering of China under Grant number SKLGIE2017-M-4-3.

Acknowledgments: This work was supported by National Natural Science Foundation of China (No. 41801319), Science and Technology Program of Henan in China (No. 182102210147), Foundation for State Key Laboratory of Geo-information Engineering of China (No. SKLGIE2017-M-4-3).

Conflicts of Interest: The authors declare no conflict of interest.

\section{References}

1. Zhang, F.; Hu, M.; Che, W.; Lin, H.; Fang, C. Framework for Virtual Cognitive Experiment in Virtual Geographic Environments. ISPRS Int. J. Geo Inf. 2018, 7, 36. [CrossRef]

2. You, L.; Lin, H. A Conceptual Framework for Virtual Geographic Environments Knowledge Engineering. ISPRS Int. Arch. Photogramm. Remote Sens. Spat. Inf. Sci. 2016, 41, 357-360. 
3. Lin, H.; Zhang, C.X.; Chen, M.; Zheng, X.Q. On virtual geographic environments for geographic knowledge representation and sharing. J. Remote Sens. 2016, 20, 1290-1298, (In Chinese with English Abstract). [CrossRef]

4. Lü, G.N. Geographic analysis-oriented virtual geographic environment: Framework, structure and functions. Sci. China Earth Sci. 2011, 54, 733-743. [CrossRef]

5. Jia, F.L.; Zhang, W.W.; Xiong, Y. Cognitive research framework of virtual geographic environment. J. Remote Sens. 2015, 19, 179-187, (In Chinese with English Abstract). [CrossRef]

6. Jiang, B.; Xiong, Y.; Qing, X.I. Voxels and the Construction of a Virtual Geographic Environment; Geomatics and Information Science of Wuhan University: Wuhan, China, 2013; Volume 38, pp. 875-878, (In Chinese with English Abstract).

7. You, L.; Lin, H. Towards a research agenda for knowledge engineering of virtual geographical environments. Ann. GIS 2016, 22, 1-9. [CrossRef]

8. Mekni, M.; Environnement, E.T.; Moulin, B. Informed virtual geographic environments for knowledge representation and reasoning in multiagent geosimulations. In Proceedings of the Third International Conference on Advanced Cognitive Technologies and Applications, Rome, Italy, 25-30 September 2011.

9. Mekni, M. Spatial Cognitive Simulation Using Informed Virtual Geographic Environments. Int. J. Comput. Netw. Commun. Secur. 2016, 4, 242.

10. Mekni, M. Spatial simulation using abstraction of virtual geographic environments. Int. J. Digit. Earth 2018, 11, 334-355. [CrossRef]

11. Lin, H.; Chen, M. Managing and sharing geographic knowledge in virtual geographic environments (VGEs). Ann. GIS 2015, 21, 261-263. [CrossRef]

12. Laurini, R. A conceptual framework for geographic knowledge engineering. J. Vis. Lang. Comput. 2014, 25, 2-19. [CrossRef]

13. Laurini, R.; Favetta, F. About External Geographic Information and Knowledge in Smart Cities. In Proceedings of the 2nd International Conference on Smart Data and Smart Cities, Puebla, Mexico, 4-6 October 2017.

14. Mekni, M.; Moulin, B. Informed Virtual Geographic Environments: A Geometrically Precise and Semantically Enriched Model for Multi-Agent Geo-Simulations. In Proceedings of the Winter Simulation Conference, Phoenix, AZ, USA, 11-14 December 2011; pp. 381-392.

15. Mekni, M. Using GIS Data to Build Informed Virtual Geographic Environments (IVGE). J. Geogr. Inf. Syst. 2013, 5, 548-558. [CrossRef]

16. Qi, G.L.; Gao, H.; Wu, T.X. The research advances of knowledge graph. Technol. Intell. Eng. 2017, 3, 4-25. [CrossRef]

17. Sowa, J.F. Principles of Semantic Networks: Exploration in the Representation of Knowledge. In Frame Problem in Artificial Intelligence; Morgan Kaufmann: Burlington, MA, USA, 1991; pp. 135-157.

18. Bollacker, K.; Cook, R.; Tufts, P. Freebase: A Shared Database of Structured General Human Knowledge. In Proceedings of the AAAI-07, Senior Member Papers Track, Vancouver, BC, Canada, 22-26 July 2007; Volume 7, pp. 1962-1963.

19. Auer, S.; Bizer, C.; Kobilarov, G.; Lehmann, J.; Cyganiak, R.; Ives, Z. DBpedia: A Nucleus for a Web of Open Data. In Proceedings of the 6th International Semantic Web Conference, Busan, Korea, 11-15 November 2007; pp. 722-735.

20. Suchanek, F.M.; Kasneci, G.; Weikum, G. Yago: A Core of Semantic Knowledge. In Proceedings of the 16th International Conference on World Wide Web, Banff, AB, Canada, 8-12 May 2007; pp. 697-706. [CrossRef]

21. Niu, X.; Sun, X.; Wang, H.; Rong, S.; Qi, G.; Yu, Y. Zhishi. Me-Weaving Chinese Linking Open Data. In Proceedings of the Information Technology in Bio- and Medical Informatics, Toulouse, France, 31 August-1 September 2011; Volume 7032, pp. 205-220.

22. Xu, B.; Xu, Y.; Liang, J.; Xie, C.; Liang, B.; Cui, W.; Xiao, Y.; Benferhat, S.; Tabia, K.; Ali, M. CN-DBpedia: A Never-Ending Chinese Knowledge Extraction System. In Lecture Notes in Computer Science; Springer: Berlin/Heidelberg, Germany, 2017; Volume 10351, pp. 428-438.

23. Schiff, S. Know It All. Available online: https:www.newyorker.com/printables/fact/060731fa_fact (accessed on 31 July 2006).

24. Yates, A.; Cafarella, M.; Banko, M.; Etzioni, O.; Broadhead, M.; Soderland, S. Textrunner: Open Information Extraction on the Web. In Proceedings of the Human Language Technologies: The Annual Conference of the North American Chapter of the Association for Computational Linguistics: Demonstrations, Rochester, NY, USA, 22-27 April 2007; pp. 25-26. 
25. Carlson, A.; Betteridge, J.; Kisiel, B.; Settles, B.; Hruschka, E.R.; Mitchell, T.M. Toward an Architecture for Never-Ending Language Learning. In Proceedings of the Twenty-Fourth AAAI Conference on Artificial Intelligence, Atlanta, GA, USA, 11-15 July 2010; Volume 5, pp. 1306-1313.

26. Wu, T.; Qi, G.; Li, C.; Wang, M. A Survey of Techniques for Constructing Chinese Knowledge Graphs and Their Applications. Sustainability 2018, 10, 3245. [CrossRef]

27. Ballatore, A.; Bertolotto, M.; Wilson, D.C. Geographic knowledge extraction and semantic similarity in OpenStreetMap. Knowl. Inf. Syst. 2013, 37, 61-81. [CrossRef]

28. Lu, F.; Yu, L.; Qiu, P.Y. On geographic knowledge graph. Int. J. Geo Inf. Sci. 2017, 19, 723-734. [CrossRef]

29. Marrero, M.; Urbano, J.; Sánchez-Cuadrado, S.; Morato, J.; Gómez-Berbís, J.M. Named entity recognition: Fallacies, challenges and opportunities. Comput. Stand. Interfaces 2013, 35, 482-489. [CrossRef]

30. Clare, D. Reading Geography between the Lines: Extracting Local Place Knowledge from Text. In Spatial Information Theory; Springer: Berlin/Heidelberg, Germany, 2013; pp. 320-337.

31. Florian, A.T.; Philip, D.S.; Christopher, B.J. Mining the Web to Detect Place Names. In Proceedings of the 2nd International Workshop on Geographic Information Retrieval, Napa Valley, CA, USA, 29-30 October 2008; pp. 43-44.

32. Yu, L.; Lu, F.; Liu, X.L. A bootstrapping based approach for open geo-entity relation extraction. Acta Geod. Cartogr. Sin. 2016, 459, 616-622. [CrossRef]

33. Loglisci, C.; Ienco, D.; Roche, M.; Teisseire, M.; Malerba, D. Toward Geographic Information Harvesting: Extraction of Spatial Relational Facts from Web Documents. In Proceedings of the 12th International Conference on Data Mining Workshops, Brussels, Belgium, 10 December 2012; pp. 789-796.

34. Kyzirakos, K.; Karpathiotakis, M.; Koubarakis, M. Strabon: A Semantic Geospatial DBMS. In Proceedings of the Information Technology in Bio-and Medical Informatics, Vienna, Austria, 4-5 September 2012; Springer: Berlin/Heidelberg, Germany, 2012; Volume 7649, pp. 295-311.

35. Duan, H.; Meng, L.; Huang, C.; Li, Y.; Li, J. A method for geo semantic spatial index on SPARQL query. Acta Geod. Cartogr. Sin. 2014, 43, 193-199. [CrossRef]

36. Gür, N.; Pedersen, T.B.; Zimányi, E.; Hose, K. A foundation for spatial data warehouses on the semantic web. Semant. Web 2017, 9, 1-31. [CrossRef]

37. Gao, Y.; Gao, S.; Li, R.; Liu, Y. A semantic geographical knowledge wiki system mashed up with Google Maps. Sci. China Ser. E Technol. Sci. 2010, 53, 52-60. [CrossRef]

38. Liu, Y.; Li, R.; Chen, K.; Yuan, Y.; Huang, L.; Yu, H. KIDGS: A geographical knowledge-informed digital gazetteer service. In Proceedings of the 2009 17th International Conference on Geoinformatics, Fairfax, VA, USA, 12-14 August 2009.

39. Qu, Y.; Liu, J.; Kang, L.; Shi, Q.; Ye, D. Question Answering over Freebase via Attentive RNN with Similarity Matrix based CNN. arXiv 2018, arXiv:1804.03317.

40. Bordes, A.; Weston, J.; Collobert, R.; Bengio, Y. Learning structured embeddings of knowledge bases. In Proceedings of the AAAI Conference on Artificial Intelligence, AAAI 2011, San Francisco, CA, USA, 7-11 August 2011.

41. Bordes, A.; Glorot, X.; Weston, J.; Bengio, Y. A semantic matching energy function for learning with multi-relational data. Mach. Learn. 2014, 94, 233-259. [CrossRef]

42. Jenatton, R.; Roux, N.L.; Bordes, A.; Obozinski, G. A latent factor model for highly multi-relational data. In Proceedings of the 25th International Conference on Neural Information Processing Systems, Lake Tahoe, NV, USA, 3-6 December 2012.

43. Socher, R.; Chen, D.; Manning, C.D.; Ng, A.Y. Reasoning with neural tensor networks for knowledge base completion. In Proceedings of the 25th International Conference on Neural Information Processing Systems, Lake Tahoe, NV, USA, 3-6 December 2012.

44. Bordes, A.; Usunier, N.; Garcia-Duran, A.; Weston, J.; Yakhnenko, O. Translating embeddings for modeling multi-relational data. In Proceedings of the 26th International Conference on Neural Information Processing Systems, Lake Tahoe, NV, USA, 5-10 December 2013; pp. 2787-2795.

45. Wang, Z.; Zhang, J.W.; Feng, J.L.; Chen, Z. Knowledge graph embedding by translating on hyperplanes. In Proceedings of the 28th AAAI Conference on Artificial Intelligence, Quebec City, QC, Canada, 27-31 July 2014; pp. 1112-1119. 
46. Lin, Y.; Zhang, J.; Liu, Z.; Sun, M.; Liu, Y.; Zhu, X. Learning entity and relation embeddings for knowledge graph completion. In Proceedings of the 29th AAAI Conference on Artificial Intelligence, Austin, TX, USA, 25-30 January 2015; pp. 2181-2187. [CrossRef]

47. Ji, G.L.; He, S.Z.; Xu, L.H.; Liu, K.; Zhao, J. Knowledge graph embedding via dynamic mapping matrix. In Proceedings of the 53rd Annual Meeting of the Association for Computational Linguistics, Beijing, China, 26-31 July 2015; pp. 687-696. [CrossRef]

48. Blanco, R.; Ottaviano, G.; Meij, E. Fast and space-efficient entity linking for queries. In Proceedings of the Eighth ACM International Conference on Web Search and Data Mining, Shanghai, China, 2-6 February 2015; pp. 179-188. [CrossRef]

49. Zhang, W.; Sim, Y.C.; Su, J.; Tan, C.L. Entity linking with effective acronym expansion, instance selection, and topic modeling. In Proceedings of the Twenty-Second international joint conference on Artificial Intelligence, Barcelona, Spain, 16-22 July 2011; pp. 1909-1914.

50. Alhelbawy, A.; Gaizauskas, R. Graph ranking for collective named entity disambiguation. In Proceedings of the 52nd Annual Meeting of the Association for Computational Linguistics (Volume 2: Short Papers), Baltimore, MD, USA, 22-27 June 2014.

51. Huang, H.; Heck, L.; Ji, H. Leveraging Deep Neural Networks and Knowledge Graphs for Entity Disambiguation. arXiv 2015, arXiv:1504.07678.

52. Zhou, B.; Sun, C.; Lin, L. LSTM based question answering for large scale knowledge base. Acta Sci. Nat. Univ. Pekin. 2018, 54, 286-292. [CrossRef]

(C) 2019 by the authors. Licensee MDPI, Basel, Switzerland. This article is an open access article distributed under the terms and conditions of the Creative Commons Attribution (CC BY) license (http://creativecommons.org/licenses/by/4.0/). 